\title{
Decreased Frequency But Not Amplitude of Quantal Synaptic Responses Associated with Expression of Corticostriatal Long-Term Depression
}

\author{
Sukwoo Choi ${ }^{1}$ and David M. Lovinger ${ }^{1,2}$ \\ Departments of ${ }^{1}$ Molecular Physiology and Biophysics and ${ }^{2}$ Pharmacology, Vanderbilt University School of Medicine, \\ Nashville, Tennessee 37232-0615
}

We have investigated the site of expression of striatal long-term synaptic depression (LTD) using analysis of $\mathrm{Sr}^{2+}$-induced asynchronous release of quanta from stimulated synapses. The cumulative amplitude distribution of $\mathrm{Sr}^{2+}$-induced asynchronous synaptic responses overlaps with that of miniature EPSCs (mEPSCs), suggesting that $\mathrm{Sr}^{2+}$-induced asynchronous responses are quantal. Quantal amplitude at stimulated synapses is not significantly altered after LTD induction, whereas quantal frequency decreases after LTD induction. The decrease in quantal frequency is prevented when LTD expression is blocked by dialyzing $10 \mathrm{~mm}$ EGTA into the postsynaptic neuron. Our findings are most consistent with the idea that expression of striatal LTD involves decreased neurotransmitter release with no change in quantal amplitude, despite the fact that induction of striatal LTD involves postsynaptic mechanisms.

Key words: long-term depression; striatum; cortex; synaptic plasticity; presynaptic expression; postsynaptic induction
Glutamatergic projections from the cortex are the major excitatory inputs to the striatum, a brain region important in motor and habit learning and a target for Huntington's and Parkinson's diseases (Graybiel et al., 1994; Knowlton et al., 1996; Lovinger and Tyler, 1996). High-frequency activation of corticostriatal synapses induces long-term synaptic depression (LTD) of transmission at these synapses (Calabresi et al., 1996; Lovinger and Tyler, 1996). Striatal LTD has been proposed as a cellular model for developmental and adult neuronal plasticity in the striatum (Calabresi et al., 1996; Choi and Lovinger, 1997). Induction of striatal LTD is thought to be dependent on activation of L-type calcium channels, leading to an increase in postsynaptic calcium (Calabresi et al., 1996; Choi and Lovinger, 1997), whereas expression of striatal LTD appears to involve a decrease in the probability of neurotransmitter release (Choi and Lovinger, 1997).

One approach to detect changes in presynaptic and postsynaptic function is to measure the amplitude and frequency of quantal synaptic responses (Del Castillo and Katz, 1954; Redman, 1990; Stevens, 1993). According to the quantal theory of neurotransmitter release, a change in quantal amplitude is interpreted as a change in postsynaptic function, whereas a change in quantal frequency is thought to represent a change in presynaptic neurotransmitter release. Most forms of homosynaptic long-term potentiation (LTP) and LTD including striatal LTD have been shown to be limited to stimulated synapses (Calabresi et al., 1992; Bliss and Collingridge, 1993). When examining LTP and LTD it is therefore necessary to measure quantal synaptic responses only from synapses expressing LTP and LTD.

$\mathrm{Sr}^{2+}$ has been shown to substitute for $\mathrm{Ca}^{2+}$ in the process of quantal release of transmitter at the neuromuscular junction, although it does so less effectively (Miledi, 1966). Furthermore,

Received May 29, 1997; revised Aug. 11, 1997; accepted Aug. 22, 1997.

Correspondence should be addressed to Dr. David M. Lovinger, Department of Molecular Physiology and Biophysics, Vanderbilt University School of Medicine, 702 Light Hall, Nashville, TN 37232-0615.

Copyright (C) 1997 Society for Neuroscience $0270-6474 / 97 / 178613-08 \$ 05.00 / 0$ the mode of action of $\mathrm{Sr}^{2+}$ is qualitatively similar to that of $\mathrm{Ca}^{2+}$, such that $\mathrm{Sr}^{2+}$ substitution does not appear to change the minimal synaptic delay in response to nerve stimulation or the standard cooperativity of transmitter release (Miledi, 1966; Meiri and Rahamimoff, 1971). However, $\mathrm{Sr}^{2+}$ reduces the efficacy of a fast, synchronous component of release and greatly facilitates a slow, asynchronous component of release, which has been shown to be quantal (Dodge et al., 1969). Recently, these observations at the neuromuscular junction have been reproduced in hippocampal cultures and slices (Goda and Stevens, 1996; Oliet et al., 1996). Analysis of asynchronous quanta have revealed that both presynaptic and postsynaptic changes are associated with expression of LTP and LTD at CA3-CA1 synapses in the hippocampus (Oliet et al., 1996, 1997). Also, $\mathrm{Sr}^{2+}$ has been shown to substitute for $\mathrm{Ca}^{2+}$ in inducing and maintaining hippocampal LTD (Goda and Stevens, 1996). These findings suggest that quantal analysis at synapses expressing LTP and LTD can be accomplished by examining $\mathrm{Sr}^{2+}$-induced asynchronous quantal release. Furthermore, it is greatly advantageous to measure $\mathrm{Sr}^{2+}$-induced asynchronous synaptic responses generated by afferent stimulation, because expression of homosynaptic LTP and LTD, including striatal LTD, is limited to synapses made by the stimulated afferents.

Although striatal LTD has been shown to involve a decrease in the probability of neurotransmitter release (Choi and Lovinger, 1997), it is not known whether there is a postsynaptic contribution to LTD expression. In the present study, we have used analysis of asynchronous quanta in the presence of $\mathrm{Sr}^{2+}$ to determine the site of expression of striatal LTD. Our findings suggest predominant presynaptic expression of LTD at corticostriatal synapses, although induction of striatal LTD requires postsynaptic mechanisms.

\section{MATERIALS AND METHODS}

Brain slices were prepared from 10- to 19-d-old rats as described previously (Choi and Lovinger, 1997). Rats were killed by decapitation, and 
the brains were cooled in ice-cold, modified artificial CSF (aCSF) containing (in $\mathrm{mm}$ ): 194 sucrose, $30 \mathrm{NaCl}, 4.5 \mathrm{KCl}, 1 \mathrm{MgCl}_{2}, 26 \mathrm{NaHCO}_{3}$, $1.2 \mathrm{NaH}_{2} \mathrm{PO}_{4}$, and $10 \mathrm{D}$-glucose adjusted to $\mathrm{pH} 7.4$ by bubbling with $95 \%$ $\mathrm{O}_{2} / 5 \% \mathrm{CO}_{2}$. Coronal sections (400 $\mu \mathrm{M}$ thick) were cut in ice-cold, modified aCSF using a manual vibroslice (World Precision Instruments, New Haven, CT). Slices were then transferred to a nylon net submerged in normal aCSF containing (in $\mathrm{mM}$ ): $124 \mathrm{NaCl}, 4.5 \mathrm{KCl}, 2 \mathrm{CaCl}_{2}, 1$ $\mathrm{MgCl}_{2}, 26 \mathrm{NaHCO}_{3}, 1.2 \mathrm{NaH}_{2} \mathrm{PO}_{4}, 10$ D-glucose, and $25 \mu \mathrm{M} \mathrm{APV}$ oxygenated and maintained at $\mathrm{pH} 7.4$ by bubbling with $95 \% \mathrm{O}_{2} / 5 \% \mathrm{CO}_{2}$ at room temperature $\left(21-24^{\circ} \mathrm{C}\right)$. A hemislice containing the cortex and striatum just anterior to the globus pallidus was completely submerged in a Plexiglas recording chamber and continuously superfused with aCSF in which $3 \mathrm{mM} \mathrm{Sr}^{2+}\left(\right.$ or $\left.\mathrm{Ca}^{2+}\right)$ and $5 \mathrm{mM} \mathrm{Mg}^{2+}$ were substituted for $2 \mathrm{mM}$ $\mathrm{Ca}^{2+}$ and $1 \mathrm{mM} \mathrm{Mg}^{2+}$. Picrotoxin and APV (25 $\mu \mathrm{M}$ each) were continuously present in the recording solution to prevent possible contamination by $\mathrm{GABA}_{\mathrm{A}}$ and NMDA receptor-mediated synaptic responses.

Whole-cell voltage-clamp recordings were made using an Axopatch 1-D amplifier (Axon Instruments, Foster City, CA). Recordings were obtained using pipettes pulled on a Flaming-Brown micropipette puller (Sutter Instrument Corp., Novato, CA). Pipette resistances ranged from 1 to $3 \mathrm{M} \Omega$ when filled with internal solution containing (in $\mathrm{mM}$ ): 120 $\mathrm{CsMeSO}_{3}, 5 \mathrm{NaCl}, 10 \mathrm{TEA}$ chloride, $10 \mathrm{HEPES}, 3-5 \mathrm{QX}-314$ (Br salt), 1.1 EGTA, 4 ATP $\left(\mathrm{Mg}^{2+}\right.$ salt), and $0.3 \mathrm{GTP}$ (Na salt), $\mathrm{pH}$ adjusted to 7.2 with $\mathrm{CsOH}$, osmolarity adjusted to $297-300 \mathrm{mmol} / \mathrm{kg}$ with sucrose. Recordings were made under differential interference contrast (DIC)enhanced visual guidance from neurons three to four cell layers below the surface of $400-\mu \mathrm{m}$-thick slices at $31 \pm 1^{\circ} \mathrm{C}$. Cells were voltageclamped at -50 to $-70 \mathrm{mV}$ during test periods before and after LTD induction. To evoke synaptic currents, stimuli were delivered through bipolar tungsten electrodes placed in the white matter dorsal to the striatum. The series resistance, which was not compensated and was typically between 3 and $10 \mathrm{M} \Omega$, was monitored continuously. Solutions were delivered to slices via superfusion driven by gravity flow. The flow rate was $2-3 \mathrm{ml} / \mathrm{min}$.

LTD was induced by pairing high-frequency stimulation (HFS, four, 1 sec duration, $100 \mathrm{~Hz}$ trains delivered one/10 sec) with $1 \mathrm{sec}$ depolarization of the postsynaptic neuron to $0 \mathrm{mV}$. For LTD experiments, neurons were initially patch-clamped in $\mathrm{Sr}^{2+}$-containing aCSF. To evoke synchronous EPSCs in the presence of $\mathrm{Ca}^{2+}$, stimuli were given at a frequency of 0.05 or $0.033 \mathrm{~Hz}$. To acquire asynchronous synaptic responses, cortical afferents were stimulated at a frequency of 0.1 or $0.2 \mathrm{~Hz}$ in the presence of $\mathrm{Sr}^{2+}$. The frequency of $\mathrm{Sr}^{2+}$-induced asynchronous synaptic responses did not change over the recording time during 0.1 or $0.2 \mathrm{~Hz}$ stimulation (data not shown). Asynchronous events were measured during a $400 \mathrm{msec}$ period beginning 30-50 msec after stimulation to eliminate synchronous synaptic responses. The small synchronous synaptic responses evoked in $\mathrm{Sr}^{2+}$ were measured and averaged including synaptic failures using pClamp version 6.0 software (Axon Instruments). Amplified currents were filtered at $1 \mathrm{KHz}$ and stored on videotape. For data analysis, whole-cell currents on videotape were digitized at up to $10 \mathrm{KHz}$ and stored on a Pentium microcomputer (Dell Computer Corp., Austin, TX). Quantal events were detected and analyzed using Mini version 1.4 as described previously (Tyler and Lovinger, 1995). The number of events used to construct cumulative histograms ranged from 125 to 1230 . Data for cumulative EPSC histograms were compared statistically using the Kolmogorov-Smirnov test. All averaged values are given as mean \pm SEM and were compared statistically by repeated measures or paired Student's $t$ test. The statistical criterion for significance was $p<0.05$.

\section{RESULTS}

\section{Characterization of $\mathrm{Sr}^{2+}$-induced asynchronous release}

We examined the effect of substituting $\mathrm{Sr}^{2+}$ for $\mathrm{Ca}^{2+}(3 \mathrm{~mm})$ on synaptic transmission at corticostriatal synapses. Stimulation of cortical afferents in the presence of extracellular $\mathrm{Ca}^{2+}$ produced fast synchronous EPSCs with few synaptic events during the 400 msec period after the end of synchronous responses. Substituting $\mathrm{Ca}^{2+}$ with $\mathrm{Sr}^{2+}(3 \mathrm{~mm})$ led to a decrease in the amplitude of synchronous EPSCs and to the appearance of asynchronous synaptic events (Fig. 1 $A, a, b)$.

Both TTX $(0.7 \mu \mathrm{M})$ and DNQX $(20 \mu \mathrm{M})$ completely blocked
$\mathrm{Sr}^{2+}$-induced asynchronous synaptic responses $(n=4$, respectively), suggesting that asynchronous synaptic responses evoked in the presence of $\mathrm{Sr}^{2+}$ are mediated by afferent activation, leading to glutamate release and activation of AMPA/kainate receptors (Fig. $1 A, c, d$ ). In addition, spontaneous EPSCs (sEPSCs) recorded in the presence of $\mathrm{Sr}^{2+}$ were completely blocked by $20 \mu \mathrm{M}$ DNQX ( $n=4$; data not shown).

Other than increasing the incidence of stimulus-evoked asynchronous EPSCs, $\mathrm{Sr}^{2+}$ did not alter any aspects of either presynaptic or postsynaptic functions. We compared the cumulative amplitude and interval distribution of sEPSCs in the presence of $\mathrm{Sr}^{2+}$ with those in the presence of $\mathrm{Ca}^{2+}$. As shown in Figure $1 B$, the cumulative amplitudes of sEPSCs in the presence of $\mathrm{Sr}^{2+}$ were not significantly different from those in the presence of $\mathrm{Ca}^{2+}$ in four of five neurons examined $(p>0.1)$. Also, the cumulative interval distribution of sEPSCs in the presence of $\mathrm{Sr}^{2+}$ was not significantly different from that in the presence of $\mathrm{Ca}^{2+}$ in three of five neurons $(p>0.05)$. Furthermore, no significant changes in the average frequency, amplitude, rise, and half-decay time of sEPSCs were observed in $\mathrm{Sr}^{2+}$ compared with $\mathrm{Ca}^{2+}(n=5 ; p>0.1)$. These findings suggest that $\mathrm{Sr}^{2+}$ does not significantly change presynaptic or postsynaptic function in the absence of afferent stimulation, and that the predominant effect of $\mathrm{Sr}^{2+}$ is to promote asynchronous neurotransmitter release.

To determine whether $\mathrm{Sr}^{2+}$-induced asynchronous synaptic responses are quantal, we compared the amplitude distribution of asynchronous synaptic responses evoked in the presence of $\mathrm{Sr}^{2+}$ with that of mEPSCs recorded in the same neuron in the presence of $0.7 \mu \mathrm{M}$ TTX (Fig. $1 C$ ). The amplitude distribution of asynchronous synaptic responses was not different from that of mEPSCs in three of four cells examined $(p>0.05)$. Furthermore, the average amplitudes, rise times, and half-decay times of asynchronous synaptic responses in the presence of $\mathrm{Sr}^{2+}$ were not significantly different from those of mEPSCs $(n=4 ; p>0.1)$. Consistent with previous observations (Oliet et al., 1996), our data suggest that asynchronous synaptic responses evoked in the presence of $\mathrm{Sr}^{2+}$ are quantal.

\section{Modulation of quantal frequency and amplitude by presynaptic and postsynaptic manipulations}

To test our ability to distinguish presynaptic and postsynaptic changes using $\mathrm{Sr}^{2+}$-induced quantal release, we examined effects of several manipulations that were designed to alter presynaptic and postsynaptic function. As shown in Figure $2 A$, applying paired stimuli [paired plus facilitation (PPF); $20 \mathrm{msec}$ interstimulus interval], a manipulation that increases the probability of neurotransmitter release, resulted in a significant increase in the average frequency of asynchronous synaptic responses. Asynchronous EPSC frequency increased to $218 \pm 37 \%$ of the response to single stimuli $(n=4 ; p<0.05)$. PPF did not produce any significant changes in the cumulative amplitude distribution of asynchronous synaptic responses in three of four neurons examined $(p>0.05)$. The average amplitudes, rise times, and half-decay times of asynchronous synaptic responses were not significantly altered by PPF $(n=4 ; p>0.1)$. We also measured synchronous synaptic responses evoked in the presence of $\mathrm{Sr}^{2+}$ in the same population of neurons in which asynchronous synaptic responses were acquired. The average amplitude of synchronous synaptic responses evoked in $\mathrm{Sr}^{2+}$ in response to the second of the paired stimuli was increased to $191 \pm 18 \%$ of the response to single stimuli.

Decreasing stimulus intensity, a manipulation that reduces the effective number of release sites, produced a significant decrease 
A

a

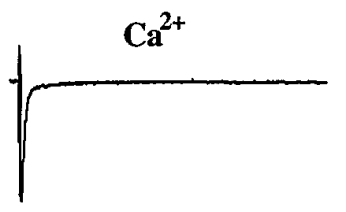

b

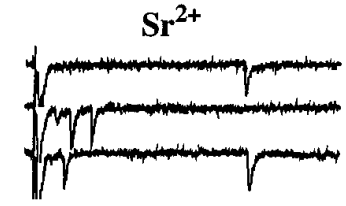

C

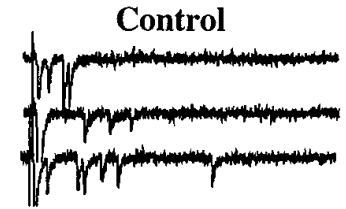

d

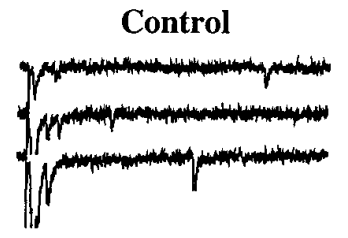

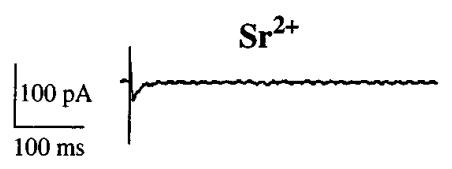
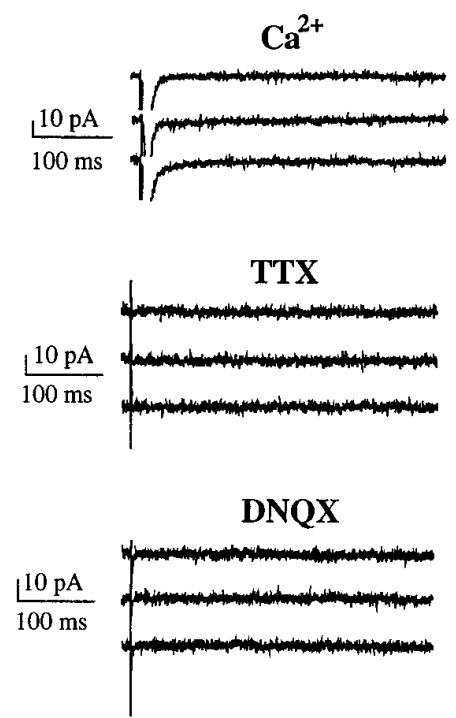
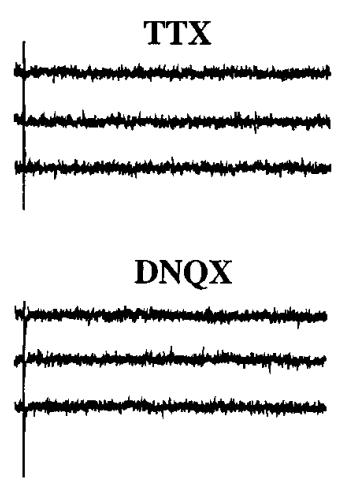

B

a

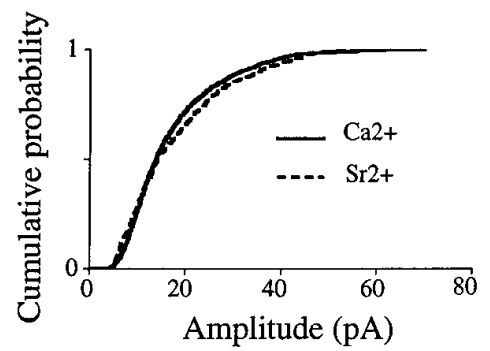

b

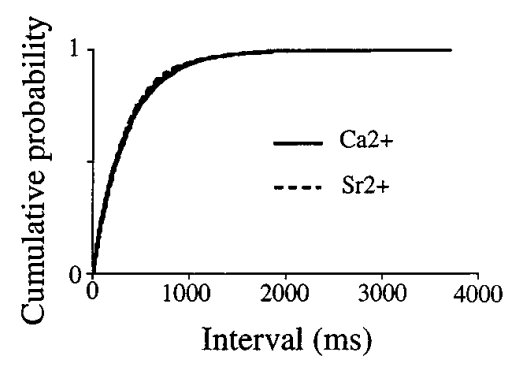

c

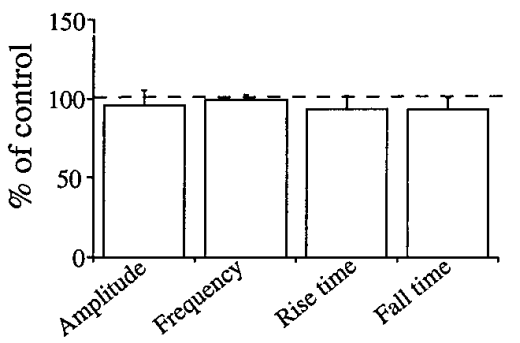

Figure 1. $\mathrm{Sr}^{2+}$ substitution for $\mathrm{Ca}^{2+}$ enhances asynchronous quantal synaptic responses mediated by AMPA/kainate receptors. $A, a$, EPSCs evoked in the presence of $\mathrm{Ca}^{2+}$ or $\mathrm{Sr}^{2+}$. Traces are averages of 20 EPSCs. Note that synchronous synaptic responses were decreased in the presence of $\mathrm{Sr}^{2+}$. $b$, EPSCs evoked in the presence of $\mathrm{Sr}^{2+}$ or $\mathrm{Ca}^{2+}$. Note the appearance of asynchronous synaptic responses in the presence of $3 \mathrm{~mm} \mathrm{Sr}{ }^{2+}$. The peaks of synchronous responses in the presence of $\mathrm{Ca}^{2+}$ are clipped off because of expansion of the current scale. $c$, EPSCs evoked in Sr ${ }^{2+}$ in the absence or presence of $0.7 \mu \mathrm{M}$ TTX. $d$, EPSCs evoked in $\mathrm{Sr}^{2+}$ in the absence or presence of $20 \mu \mathrm{M}$ DNQX. $B, a, b$, Comparison of the cumulative amplitude distribution $(a)$ and interval distribution $(b)$ of sEPSCs in the presence of $\mathrm{Sr}^{2+}$ with those in the presence of $\mathrm{Ca}^{2+}$. $c$, Comparison of the average amplitude, frequency, rise time, and half-decay time of sEPSCs in the presence of $\mathrm{Sr}^{2+}$ with those in the presence of $\mathrm{Ca}^{2+}$. Control was sEPSCs in the presence of $\mathrm{Sr}^{2+}$. C, a Comparison of the cumulative amplitude distribution of asynchronous synaptic responses evoked in $\mathrm{Sr}^{2+}$ with that of mEPSCs recorded in the presence of $0.7 \mu \mathrm{M}$ TTX in the same neuron. $b$, Comparison of the average amplitude, rise time, and half-decay time of asynchronous synaptic responses with those of mEPSCs. Control was asynchronous synaptic responses. Asyn, Asynchronous synaptic responses.

in the average frequency of asynchronous synaptic responses to $42.7 \pm 2.1 \%$ of control $(n=5 ; p<0.001$; Fig. $2 B)$. This manipulation did not produce any significant change in the cumulative amplitude distribution in five of five neurons examined $(p>0.1)$. The average amplitudes, rise times, and half-decay times of asynchronous synaptic responses were not significantly altered by decreasing stimulus intensity $(p>0.1)$. The average amplitude of synchronous synaptic responses measured in the same individual neurons was reduced to $38.5 \pm 3.4 \%$ of control by decreasing stimulus intensity.

A postsynaptic manipulation, changing the holding potential from -60 to $-40 \mathrm{mV}$, produced a significant leftward shift of the cumulative amplitude distribution in four of five neurons exam- ined ( $p<0.0001$; Fig. $2 C$ ). The average amplitude of asynchronous synaptic responses was decreased to $66.7 \pm 3.2 \%$ by this change in holding potential $(n=5 ; p<0.001)$. However, the average frequency, rise time, and half-decay time were not significantly altered by this change in holding potential $(p>0.1)$. These findings indicate that analysis of asynchronous quanta is sensitive to changes induced both by the presynaptic and postsynaptic manipulations.

\section{Association of a decrease in quantal frequency, but not quantal amplitude, with striatal LTD}

To determine whether striatal LTD is associated with changes in presynaptic or postsynaptic functions, we measured both the 
A

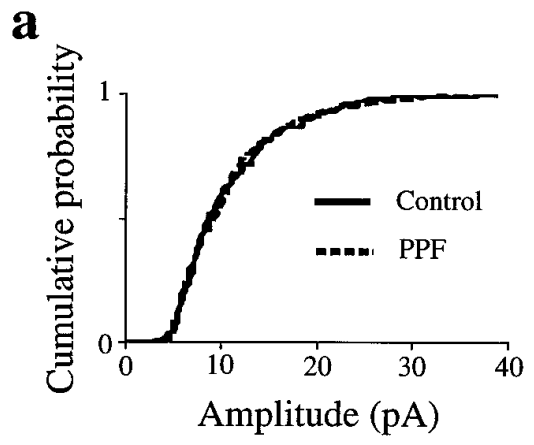

B

Figure 2. Changes in asynchronous quantal amplitude and frequency are sensitive to both presynaptic and postsynaptic manipulations. $A$, $a$, Comparison of the cumulative amplitude distribution of asynchronous synaptic responses evoked in a single neuron by single stimuli with that evoked by PPF $(20 \mathrm{msec}$ interstimulus interval). $b$, Comparison of the average amplitude, frequency, rise time, and half-decay time of asynchronous synaptic responses evoked by single stimuli with those evoked by paired stimuli. Control was asynchronous responses evoked by single stimuli. $B, a$, Comparison of cumulative amplitude distribution of asynchronous responses in a single neuron evoked by high-intensity stimulation with that by low-intensity stimulation. $b$, Comparison of the average amplitude, frequency, rise time, and half-decay time of responses evoked by high-intensity stimulation with those evoked by low stimulus intensities. Control was asynchronous synaptic responses evoked by high-intensity stimulation. $C, a$, Comparison of the cumulative amplitude distribution of asynchronous responses evoked at $-60 \mathrm{mV}$ with those evoked at $-40 \mathrm{mV} . b$, Comparison of the average amplitude, frequency, rise time, and half-decay time of asynchronous synaptic responses at $-60 \mathrm{mV}$ with those at $-40 \mathrm{mV}$. Control was asynchronous responses evoked at $-60 \mathrm{mV}$. ${ }^{*} p<0.05$. b

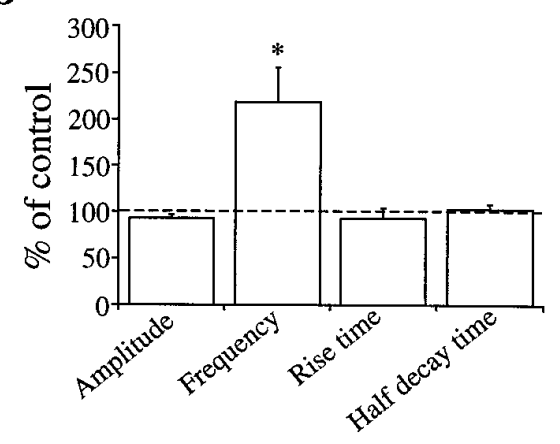

b

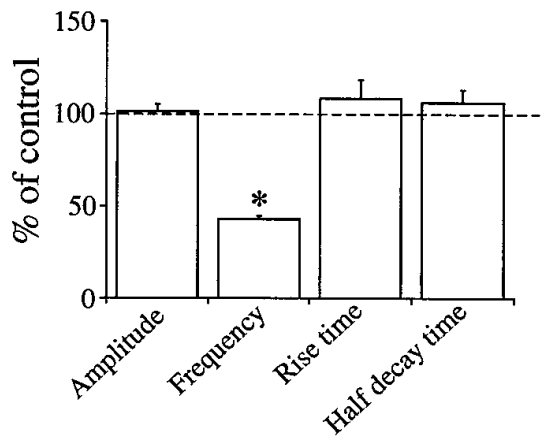

b

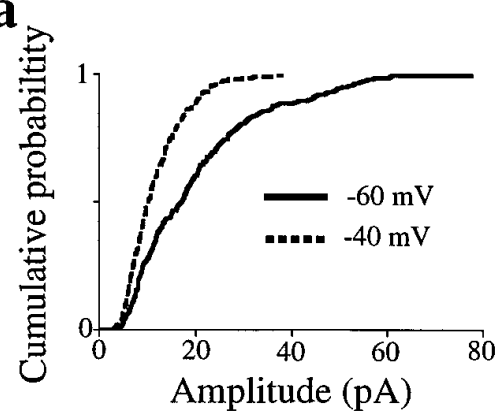

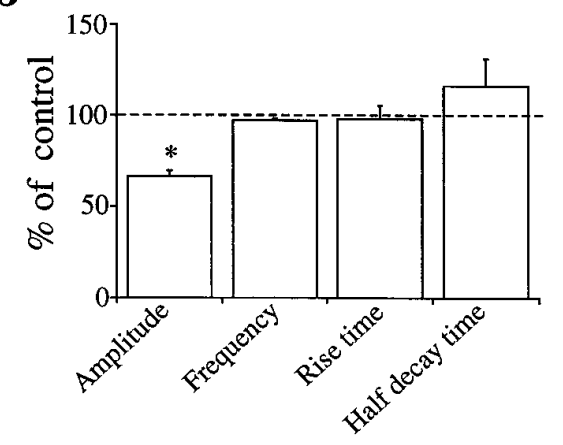

amplitude and frequency of asynchronous synaptic responses before and after LTD induction (Fig. 3). In this experiment, slices were first superfused with $\mathrm{Sr}^{2+}-\mathrm{aCSF}$ to acquire asynchronous events and then washed with $\mathrm{Ca}^{2+}-\mathrm{aCSF}$. After the EPSC amplitude stabilized (10-15 min), LTD was induced by pairing HFS with postsynaptic depolarization. After establishing that the LTD had remained stable for $15-20 \mathrm{~min}, \mathrm{Sr}^{2+}-\mathrm{aCSF}$ was reapplied, and once EPSC amplitude reached a steady level in the presence of $\mathrm{Sr}^{2+}$ (10-15 $\left.\mathrm{min}\right)$, asynchronous events were acquired. No significant changes in the cumulative amplitude distribution of asynchronous synaptic responses were detected after LTD induction in four of five cells $(p>0.05)$. Furthermore, the average amplitudes, rise times, and half-decay times of asynchronous synaptic responses were not altered after LTD induction $(p>$ 0.1 ). However, the average frequency of asynchronous synaptic responses was decreased to $64.6 \pm 4.3 \%$ of control after LTD induction $(p<0.005)$. The magnitude of the frequency decrease appeared to be sufficient to account for the magnitude of LTD, because the decrease in EPSC amplitude and the associated frequency decrease after LTD induction were similar to changes in synaptic strength and the associated frequency changes induced by PPF and decreasing stimulus intensity (compare Figs. 2, 3 ). In addition, the average amplitude of synchronous synaptic responses evoked in the presence of $\mathrm{Sr}^{2+}$ after LTD induction was decreased to $62.1 \pm 6.5 \%$ of control, which was not significantly different from the magnitude of LTD in the presence of $\mathrm{Ca}^{2+}$ (paired $t$ test, $p>0.3$ ).

Striatal LTD has been shown to be specific to the stimulated pathway (Calabresi et al., 1992). To determine whether the decrease in asynchronous quantal frequency is specific to the syn- 
A



C

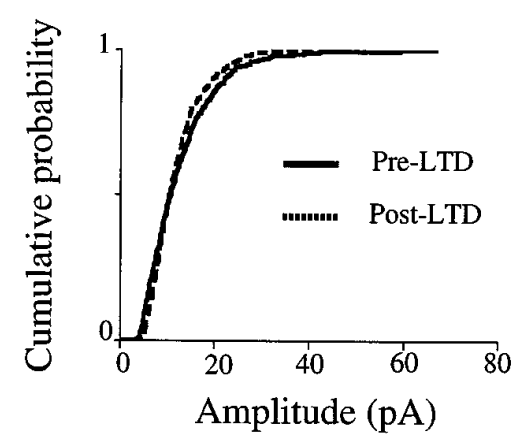

B

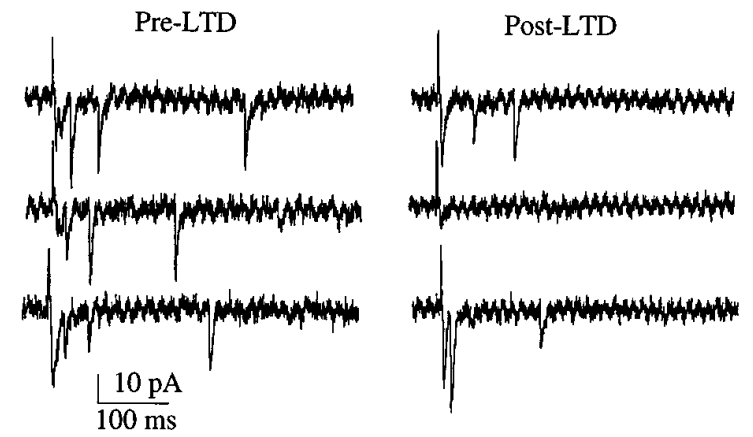

D

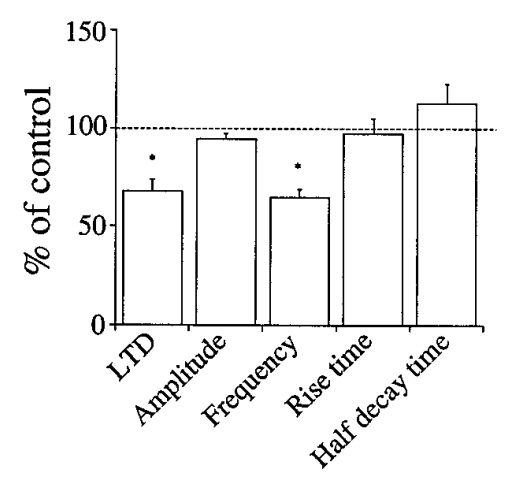

Figure 3. Striatal LTD is associated with a decrease in quantal frequency but not quantal amplitude. A, Average changes in synchronous EPSC amplitude after LTD induced by pairing HFS of cortical afferents with simultaneous depolarization of the postsynaptic neuron $(n=5)$. EPSCs were recorded in the presence of $\mathrm{Ca}^{2+} . \mathrm{Sr}^{2+}$-induced asynchronous synaptic responses were acquired in the same population of neurons before and after the period during which LTD was induced and maintained in the presence of $\mathrm{Ca}^{2+}$-containing aCSF. Points are values averaged over 1 min epochs. EPSCs shown above the graph were recorded at times indicated by letters in one of five experiments. $B$, EPSCs evoked in the presence of $\mathrm{Sr}^{2+}$ before and after LTD induction in the neuron shown in the sample traces in $A$. $C$, Comparison of the cumulative amplitude distribution of asynchronous synaptic responses evoked in the presence of $\mathrm{Sr}^{2+}$ before and after LTD induction in the neuron shown in $A$. In this particular neuron, the average frequency of asynchronous synaptic responses decreased to $60.3 \%$ of control after LTD induction. $D$, Changes in the average amplitude, frequency, rise time, and half-decay time of asynchronous synaptic responses after LTD induction. LTD at the bottom of the bar graph represents the magnitude of synaptic depression in the presence of $\mathrm{Ca}^{2+}$ after LTD induction. ${ }^{*} p<0.05$.

apses activated by afferent stimulation, we measured spontaneous synaptic events occurring outside of the $400 \mathrm{msec}$ time window of stimulation-associated events in the presence of $\mathrm{Sr}^{2+}$. We assumed that these synaptic events during the interstimulus period were primarily mEPSCs, although some events could be action potential-evoked EPSCs. We could acquire a reasonable number $(>100)$ of synaptic events during the interstimulus period in only one of five neurons (Fig. 4). In this particular neuron, the cumulative amplitude and interval distributions of synaptic events occurring during the interstimulus period were not altered after LTD induction $(p>0.05)$. The average frequency of asynchronous synaptic responses was decreased to $61.5 \%$ of control after LTD induction, whereas the cumulative amplitude distribution of asynchronous synaptic responses was not altered $(p>0.1)$. This finding suggests that synaptic events during the interstimulus period are primarily sEPSCs, and that the decrease in quantal frequency after LTD induction appears to be limited to the stimulated pathway.

LTD experiments were repeated in recordings from neurons dialyzed internally with $10 \mathrm{~mm}$ EGTA. As shown in Figure 5, Both LTD and the associated decrease in asynchronous quantal frequency were blocked by the inclusion of $10 \mathrm{~mm}$ EGTA in the pipette solution. The cumulative amplitude distribution of asynchronous synaptic responses was not altered after paired HFS and depolarization in four of four cells examined in the presence of $10 \mathrm{~mm}$ EGTA $(p>0.05)$. Also, the average amplitude, rise time, and half-decay time of asynchronous synaptic responses were not altered under these conditions. These findings suggest that the decrease in quantal frequency is strongly associated with LTD expression.

\section{DISCUSSION}

Our findings suggest that expression of striatal LTD is associated with a decrease in the frequency, but not the amplitude, of asynchronous "quantal" synaptic responses. We have previously demonstrated that LTD involves increased paired pulse facilitation and coefficient of variation of synaptic responses (Choi and Lovinger, 1997). Together, these observations suggest that stria- 


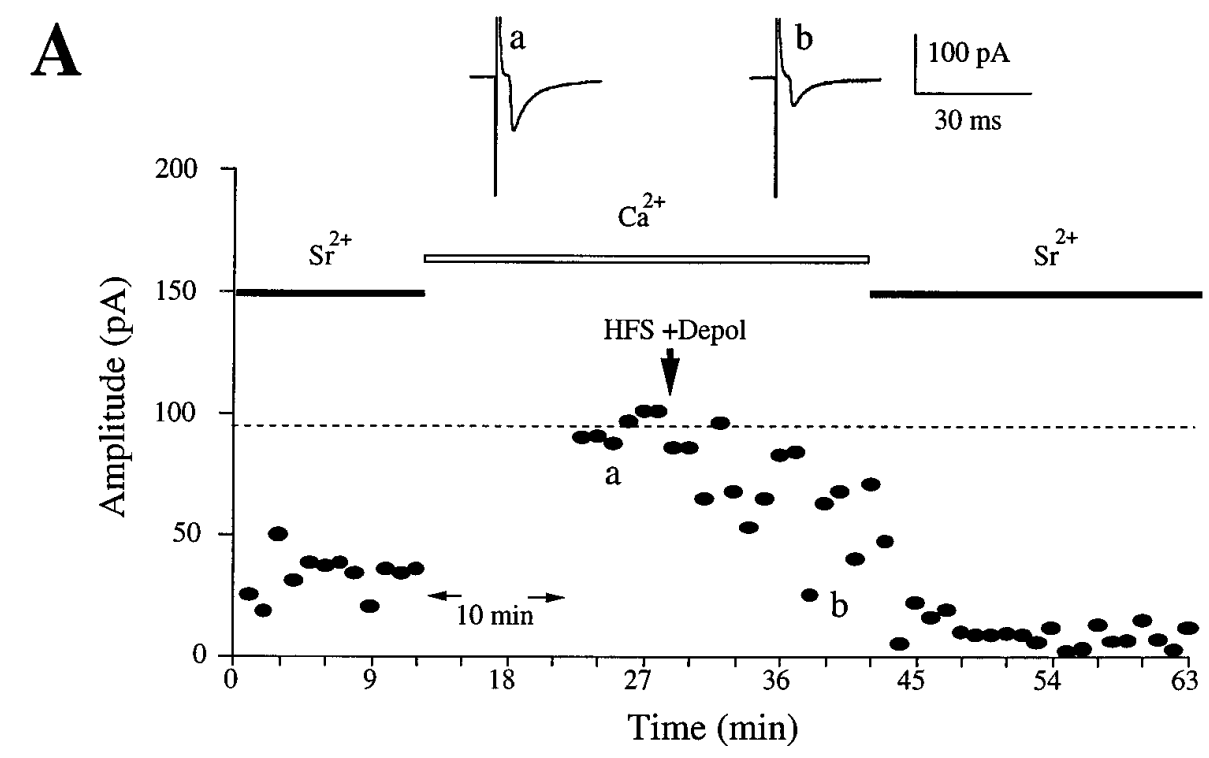

B

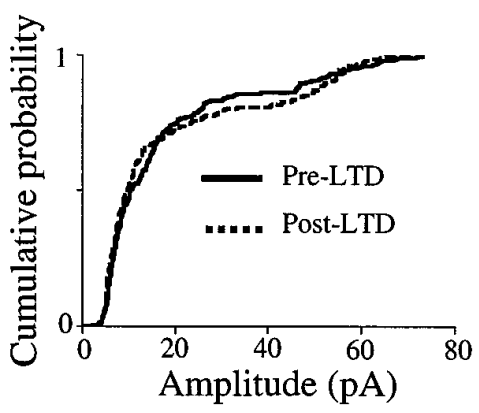

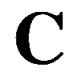

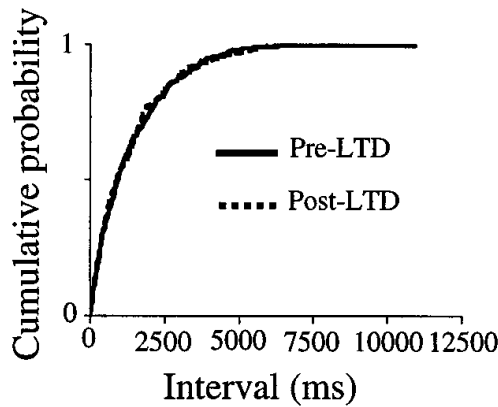

D

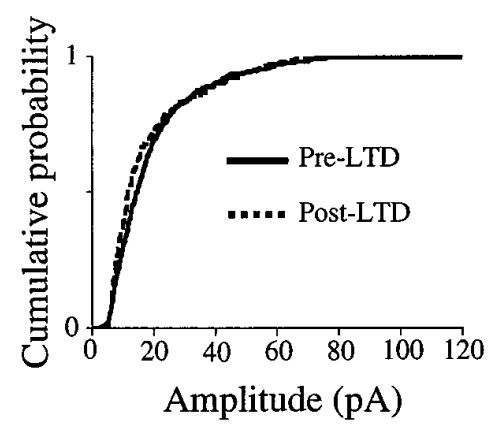

Figure 4. The decrease in quantal frequency after LTD induction is limited to stimulated synapses. $A$, Graph showing changes in the amplitude of synchronous EPSCs in the presence of $\mathrm{Sr}^{2+}$ or $\mathrm{Ca}^{2+}$. When washing a slice with $\mathrm{Ca}^{2+}$-containing aCSF $(\sim 10$ min), no afferent stimulation was used, because in our preliminary experiments, most of the whole-cell recordings appeared to be unstable with afferent stimulation during the wash of slices with $\mathrm{Ca}^{2+}$-containing aCSF. LTD was induced by pairing high-frequency stimulation with postsynaptic depolarization. Points are values averaged over 1 min epochs. EPSCs shown above the graph were recorded at times indicated by letters. Note that synchronous synaptic responses in the presence of $\mathrm{Sr}^{2+}$ were also decreased after LTD induction. $B$, Comparison of the cumulative amplitude distribution of asynchronous synaptic responses before and after LTD induction in the neuron shown in $A$. Note the lack of change in amplitude despite the fact that the average frequency of asynchronous synaptic responses was decreased to $61.5 \%$ of control after LTD induction in this neuron. $C, D$, The cumulative intervals $(C)$ and amplitudes $(D)$ of sEPSCs occurring during the interstimulus period in the presence of $\mathrm{Sr}^{2+}$ were compared before and after LTD induction in the neuron shown in $A$.

tal LTD expression most likely involves a decrease in the probability of neurotransmitter release at corticostriatal glutamatergic transmission with little change in postsynaptic responsiveness.

An alternative explanation that remains viable is a preferential loss of responsiveness at synapses with high probability of release (Pr), leading to a bias toward activation of low-Pr synapses. Such a change could occur by a complete loss of postsynaptic responsiveness at high-Pr synapses. Although LTD at central synapses has not been shown to involve an increase in the number of silent synapses, some evidence suggests that the induction of CA1 LTP involves a decrease in the number of silent synapses that may be produced by the upregulation of the number or activity of AMPA receptors (Isaac et al., 1995; Liao et al., 1995). The mechanism underlying changes in the number of silent synapses appears to be viable even for preexisting functional synapses, leading to an increase in quantal amplitude after LTP induction (Liao et al.,
1995). Consistent with these findings, changes in quantal amplitude as well as frequency associated with LTP and LTD have been detected using analysis of $\mathrm{Sr}^{2+}$-induced asynchronous quantal release in the CA1 region of hippocampus (Oliet et al., 1996, 1997). It should be noted, however, that a presynaptic expression mechanism has also been suggested for CA1 LTP and LTD (Malinow and Tsien, 1990; Bolshakov and Siegelbaum, 1994; Stevens and Wang, 1994; Kullmann and Siegelbaum, 1995). In contrast to hippocampal LTP, analysis of asynchronous quanta at corticostriatal synapses indicates that striatal LTD involves a decrease in quantal frequency without any significant change in quantal amplitude. Thus, our results differ from those expected if postsynaptic responses were decreased at all synapses. Complete loss of postsynaptic responses at a subset of synapses would have to occur to produce the changes seen with striatal LTD. Furthermore, the evidence for decreased release probability observed in 


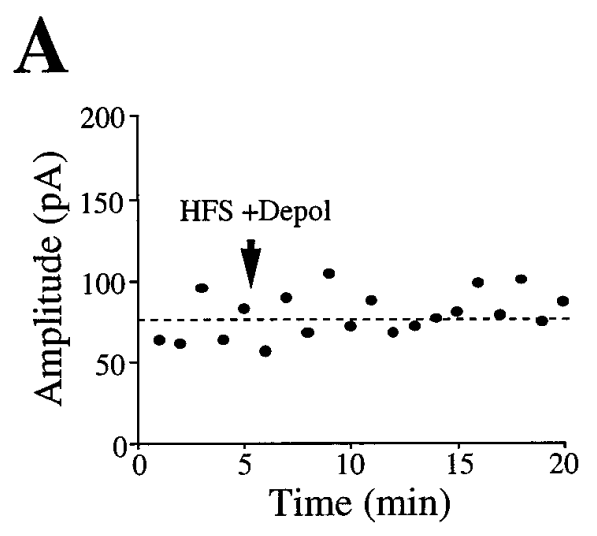

\section{B}
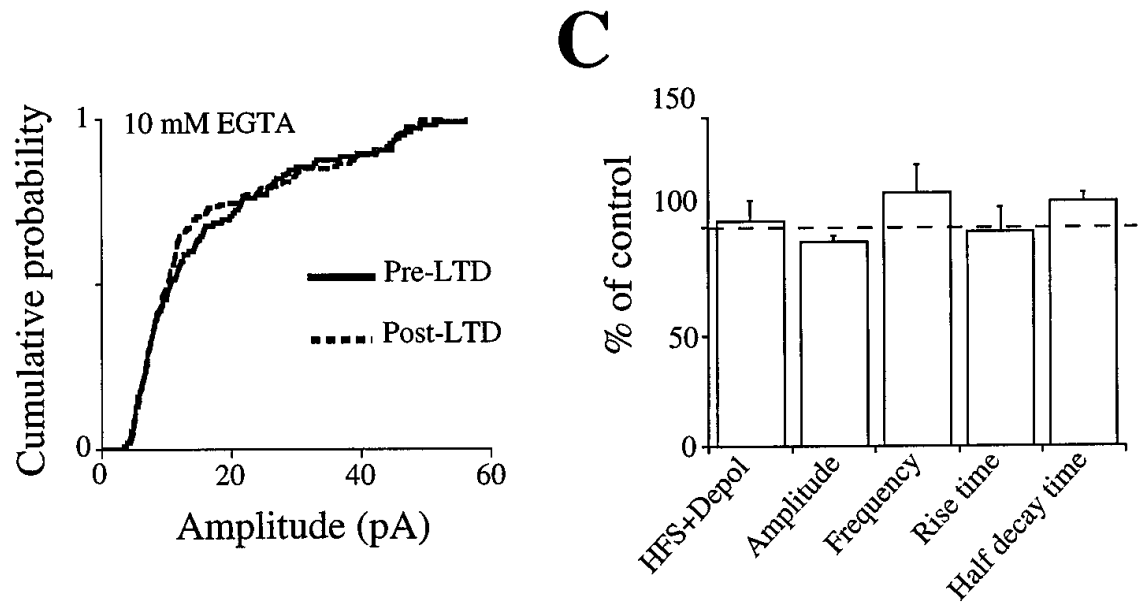

Figure 5. The decrease in quantal frequency is tightly associated with LTD expression. $A$, Paired HFS and depolarization did not produce LTD at synapses onto cells dialyzed with $10 \mathrm{~mm}$ EGTA. Points are values over $1 \mathrm{~min}$ epochs from a single neuron in $\mathrm{Ca}^{2+}$-containing aCSF. $B$, Graph comparing the cumulative amplitude distribution of asynchronous synaptic responses before and after paired HFS and depolarization in the neuron shown in $A$. In this particular neuron, EPSC amplitude in the presence of $\mathrm{Ca}^{2+}$ was $113.2 \%$ of control after paired HFS and depolarization, whereas the average frequency of asynchronous synaptic responses was $108.5 \%$ of control. $C$, Bar graph showing percent of control average amplitude, frequency, rise time, and half-decay time of asynchronous synaptic responses after paired HFS and depolarization in the cells dialyzed with 10 mM EGTA. HFS + Depol, Percent of control magnitude of synchronous synaptic responses in the presence of $\mathrm{Ca}^{2+}$ after pairing HFS with depolarization in the neurons dialyzed with 10 mM EGTA.

our previous studies (Choi and Lovinger, 1997) could only be accounted for if this postsynaptic response loss occurred solely at high-Pr synapses. Thus, although it is possible to account for the changes in transmission during striatal LTD with a modified postsynaptic synapse-silencing hypothesis, it is more likely that a presynaptic decrease in release probability is responsible for LTD expression.

Evidence suggests that one form of hippocampal LTD at CA3CA1 synapses involves postsynaptic induction and apparent presynaptic expression in neonatal and young rats (Bolshakov and Siegelbaum, 1994; Oliet et al., 1997). At present it is unclear whether this form of LTD involves a decrease in the number of release sites or the probability of neurotransmitter release. This type of LTD exhibits induction and expression mechanisms similar to that of striatal LTD; including a postsynaptic locus of induction, a lack of dependence on NMDA receptors, involvement of metabotropic glutamate receptors and voltage-gated calcium channels, and a change in quantal frequency but not amplitude associated with expression (Bolshakov and Siegelbaum, 1994; Oliet et al., 1997). Thus, mechanisms that contribute to expression of striatal LTD may also participate in LTD at excitatory synapses in other brain regions. It should be noted, however, that more than one form of LTD appears to exist in the hippocampal CA1 region (Oliet et al., 1997). A form of LTD that depends on NMDA receptor activation for its initiation can be induced at these same hippocampal synapses and appears to have a postsynaptic locus of expression (Stevens and Wang, 1994; Selig et al., 1995; Oliet et al., 1996, 1997).

One potential problem with $\mathrm{Sr}^{2+}$ substitution could be that $\mathrm{Sr}^{2+}$ may occlude some of the mechanisms underlying expression of striatal LTD. However, $\mathrm{Sr}^{2+}$ does not appear to produce any significant change in either the frequency or amplitude of sEPSCs, suggesting that it does not interfere with presynaptic or postsynaptic functions, other than promoting asynchronous release, at corticostriatal synapses. Furthermore, the magnitude of LTD measured as the decrease in synchronous EPSC amplitude in the presence of $3 \mathrm{mM} \mathrm{Sr}^{2+}$ and $5 \mathrm{mM} \mathrm{Mg}^{2+}$ was not signifi- cantly different from that in the presence of $3 \mathrm{mM} \mathrm{Ca}^{2+}$ and $5 \mathrm{~mm}$ $\mathrm{Mg}^{2+}$. Thus, the expression mechanism of striatal LTD appears to be preserved in the presence of $\mathrm{Sr}^{2+}$.

A variety of evidence indicates that induction of striatal LTD is dependent on postsynaptic mechanisms (Calabresi et al., 1996; Choi and Lovinger, 1997). If striatal LTD expression truly involves presynaptic changes, as our evidence suggests, then the molecular events underlying these presynaptic changes likely involve retrograde signals (Bliss and Collingridge, 1993).

\section{REFERENCES}

Bliss TVP, Collingridge GL (1993) A synaptic model of memory: longterm potentiation in the hippocampus. Nature 361:31-39.

Bolshakov VY, Siegelbaum SA (1994) Postsynaptic induction and presynaptic expression of hippocampal long-term depression. Science 264:1148-1152.

Calabresi P, Maj R, Pisani A, Mercuri NB, Bernardi G (1992) Longterm synaptic depression in the striatum: physiological and pharmacological characterization. J Neurosci 12:4224-4233.

Calabresi P, Pisani A, Mercuri NB, Bernardi G (1996) The corticostriatal projection: from synaptic plasticity to dysfunctions of basal ganglia. Trends Neurosci 19:19-24.

Choi S, Lovinger DM (1997) Decreased probability of neurotransmitter release underlies striatal LTD and postnatal development of corticostriatal LTD. Proc Natl Acad Sci USA 94:2665-2670.

Del Castillo J, Katz B (1954) Quantal components of the end-plate potential. J Physiol (Lond) 124:560-573.

Dodge FA, Miledi R, Rahamimoff R (1969) Strontium and quantal release of transmitter at the neuromuscular junction. J Physiol (Lond) 200:267-283.

Goda Y, Stevens CF (1996) Long-term depression properties in a simple system. Neuron 16:103-111.

Graybiel AM, Aosaki T, Flaherty AW, Kimura M (1994) The basal ganglia and adaptive motor control. Science 265:1826-1831.

Isaac JTR, Nicoll RA, Malenka RC (1995) Evidence for silent synapses: implication for the expression of LTP. Neuron 15:427-434.

Knowlton BJ, Mangels JA, Squire LR (1996) A neostriatal habit learning system in humans. Science 273:1399-1402.

Kullmann DM, Siegelbaum SA (1995) The site of expression of NMDA receptor-dependent LTP: new fuel for an old fire. Neuron 15:997-1002.

Liao D, Hessler NA, Malinow R (1995) Activation of postsynaptically silent synapses during pairing-induced LTP in CA1 region of hippocampal slice. Nature 375:400-404. 
Lovinger DM, Tyler E (1996) Synaptic transmission and modulation in the neostriatum. Int Rev Neurobiol 39:77-111.

Malinow R, Tsien RW (1990) Presynaptic enhancement shown by whole-cell recordings of long-term potentiation in hippocampal slices. Nature 346:177-180.

Meiri U, Rahamimoff R (1971) Activation of transmitter release by strontium and calcium ions at the neuromuscular junction. J Physiol (Lond) 215:709-726.

Miledi R (1966) Strontium as a substitute for calcium in the process of transmitter release at the neuromuscular junction. Nature 212:1233-1234.

Oliet SHR, Malenka RC, Nicoll RA (1996) Bidirectional control of quantal size by synaptic activity in the hippocampus. Science 271:1294-1297.
Oliet SHR, Malenka RC, Nicoll RA (1997) Two distinct forms of longterm depression coexist in CA1 pyramidal cells. Neuron 18:969-982.

Redman S (1990) Quantal analysis of synaptic potentials in neurons of the central nervous system. Physiol Rev 70:165-198.

Selig DK, Hjelmstad GO, Herron C, Nicoll RA, Malenka RC (1995) Independent mechanisms for long-term depression of AMPA and NMDA responses. Neuron 15:417-426.

Stevens CF (1993) Quantal release of neurotransmitter and long-term potentiation. Cell 72:55-63.

Stevens CF, Wang Y (1994) Changes in reliability of synaptic function as a mechanism for plasticity. Nature 371:704-707.

Tyler E, Lovinger DM (1995) Metabotropic glutamate receptor modulation of synaptic transmission in corticostriatal co-cultures: role of calcium influx. Neurophamacology 34:939-952. 